\title{
Research on Improving Reading Capability of Students in Vocational English Teaching
}

\author{
Zhang Ting \\ Urban Vocational College of Sichuan
}

Keywords: vocational English teaching; students; reading capability; strategy improvement

\begin{abstract}
Reading capability play a very important role in English learning in higher vocational colleges, not only helping students to expand their vocabulary, but also affecting the cultivation of other capabilities. Therefore, while developing English teaching, teachers should pay attention to the cultivation of students' reading capabilities. This paper is to explore effective strategies to enhance students' reading capabilities and hopefully provide relevant reference for researchers.
\end{abstract}

For some English learners, reading is difficult and also crucial. Due to the grammatical restrictions, many students can only make simple literal explanations, and they can not understand the logical relationship or grasp the intrinsic meaning from reading materials. Therefore, teachers shall focus on the learning methods and the language basics to teach and guide, cultivate students' reading habits, and enhance their vocabulary, so that their reading capabilities can be gradually improved.

\section{Current Status of Teaching of Vocational English Reading}

At present, while carrying out English teaching in higher vocational colleges, grammar translation method is commonly seen and adopted by many teachers, that is, take language as the core in classroom teaching. For example, in the process of teaching, some teachers attach great importance to the vocabulary, analyze the linguistic phenomenon and grammatical structures in the sentences, and impart the grammar language knowledge, vocabulary knowledge and pronunciation knowledge to students with the help of reading materials. It costs a great deal of time to practice, review and teach. It aims to cultivate students' capabilities of thinking and discourse, but the effectiveness of reading can be hardly guaranteed, and poor reading habits could be developed. Regardless of any type of articles, students always try to understand it word by word, and whenever they encounter a new word, they immediately consult the dictionary. They believe that reading is to understand the grammar, look up new words, and understand the meaning of the words and the sentences, which cause loss of capabilities to think independently and solve problems, and reading difficulties without reference books. In this case, it is difficult for students to improve their reading speed and fully understand the entire article, which is extremely unfavorable for their reading capabilities improvement.

\section{Lack of Certain Reading Skills}

In the process of reading materials, many students will usually analyze the grammatical knowledge of the sentences, skip or skim through new words, instead of guessing and inferring based on the meaning of similar words, which has a certain impact on the efficiency of reading. Plenty of students have poor reading habits such as whispering and reading word by word, which are extremely detrimental to students' concentration and lead to failure to capture the article theme and essence. More importantly, these will affect students' reading comprehension and speed.

An educator once said that culture covers language, meaning that learning a language well requires a good understanding of cultural norms and models. At present, many students cannot understand the articles in depth and focus on the surface work only. They hold the view that translating the article is a process of understanding the article. However, this is not the case. Lack of 
the background knowledge of culture will hinder the understanding of the article. Therefore, emphasis should be put on accumulating more cultural background knowledge.

Nowadays, due to limited teaching hours and heavy teaching tasks for teachers, the way to carry out reading training is to let the students do reading exercises and check answers afterwards. This pattern emphasizes the answers other than the students' thinking capabilities. While doing exercises, teachers do not attach importance to the construction of reading teaching situations, so the information students can use is partial, which consequently cause ignorance of implicit information and relevance, and students can not accurately judge the issues. Over time, many students will lose the enthusiasm for learning English.

\section{Solve the Existing Problems of Students' English Reading Capabilities}

Although students have mastered a basic vocabulary, they still encounter new words either in the exercise materials or in the tests. It will be difficult for them to understand the sentence with few new words. Therefore, students shall accumulate their vocabulary through various channels in the future study.

While reading the materials, lots of students are only able to understand the sentences with simple structure, and feel overwhelmed when they encounter difficult sentences or long sentences, as they do not understand the grammatical structure and know how to divide the sentence structure, which leads to lower reading efficiency.

In addition to certain grammatical knowledge and vocabulary, students shall also acquire some reading skills so that their reading capabilities can be progressively improved. However, from a practical point of view, many students do not master enough reading skills, which affects not only their reading speed, but also the overall effectiveness of reading.

\section{Strategies on Improving Students' Reading Capabilities in Higher Vocational English Teaching}

Firstly, students shall be fully aware of the negative impact of incomprehension of the cultural background. Secondly, make full use of the background knowledge related to western countries in the textbooks in order to help students explore the implied cultural connotations, and in the meantime, tell students some western common sense of communication and customs as well as the habits and thinking ways of westerners. Finally, teachers may encourage students to read material that involves breaking the national cultural backdrop. By acquiring these knowledge, students can better understand the theme and cultural background of the articles, have broader cultural horizon and sensitivity to similarities and differences between Chinese and foreign cultures. More importantly, it allows students to build their own cultural knowledge system, so as to better interpret the textual semantics.

Compared to high school English learning, students bear less pressure on English learning in higher vocational colleges, so a large number of students do not pay enough attention to this course, especially in reading. Due to greater difficulty, students are reluctant to spend energy and time. Therefore, as a teacher, it is necessary to help students to develop good reading habits over their daily teaching. In the process of reading, many students read by using hands, which has an impact on not only the reading speed, but also their understanding of the article. Over time, students will lose the enthusiasm and interest in reading English articles. In response to this situation and based on the actual situation of students, teachers need to train their short essay reading, and they will gradually form a sense of language after a long-term practice. In the meantime, teachers can arrange all the students to read a same article in class. For the first time, students need to quickly read, comprehend the vocabulary, skip the new words, and grasp a basic understanding of the entire article, without considering a number of individual difficult sentences. For the second time, students can chew the meaning of the article carefully.

A lot of reading is an essential prerequisite for students to improve their reading capabilities. In order to ensure a large amount of reading for students, teachers shall stimulate students' reading 
interest and encourage them to study independently. Therefore, while developing daily teaching, teachers shall conduct individualized teaching by selecting appropriate reading materials based on each student's actual situation. For whose without a good foundation in English and weak capabilities, teachers can arrange them to read stories which are interesting but not difficult. For those with a good foundation, we can increase the difficulty of reading materials, so that their discovery and curiosity desire can be inspired. In essence, a very important reason that many students encounter reading difficulties and cannot improve reading efficiency is that they do not accumulate enough vocabulary. Therefore, teachers should attach great importance to helping students expand the vocabulary. Firstly, if the vocabulary is within the scope of teaching program, the teacher shall require the student to firmly master and learn to use flexibly. Secondly, before class, teachers need to prepare some reading materials that cover new words and grammar points and give them to students for training in reading. Over time, students will naturally build up their vocabulary. Thirdly, in the process of actual teaching, teachers can teach in English, so that students' listening capabilities and vocabulary will be improved. Finally, the method of derivation, transformation and synthesis can be recommended to students to memorize words, such as: like-dislike, con-sider-considerate-consideration-considering, through which students can accumulate a plenty of words.

Generally, there are methods of shallow reading, deep reading and repeated reading and others. Firstly, shallow reading. Shallow reading is an overall understanding of the outline and general idea of the article through skimming and extensive reading method. A general idea can be grasped by reading the article title, and then the full text can be browsed in a quick speed. Secondly, deep reading. Deep reading is to grasp the details of the plot and key points by a detailed and intensive reading method. Both the logic between sentences and structure of paragraphs need to be acquired, so that the event trends and causes in the article can be grasped, the disputes among characters can be clarified, and the meaning and intent of the article can be comprehended. Thirdly, repeat reading. After a summary and conclusion, teachers should allow students to read the full text or analyze and understand the main words and phrases so that they can understand the meaning and the ultimate purpose of the article deeply. To improve the overall efficiency of reading, the following reading skills should be emphasized.

The reading focus shall be put on a part of the article instead of the whole one. Therefore, teachers shall train students to quickly browse the original text, and then capture the key sentences and paragraphs, the trunks as well as the key words.

When reading an essay, it is inevitable to encounter new words, and it is a challenge to guess their meaning for both the students who have solid vocabulary and those with weak knowledge in English. However, not every new word needs to be focused and understood. How to grasp the key words in the article and guess the meaning deserves a speculation by teachers. In practice, teachers can implement the following specific guessing training. First, focus their attention on verbs in sentences. This is because many of the adverbs and adjectives in the article do not affect the comprehension of the overall meaning. In the meantime, students shall be instructed to sort out the new words that do not have much meaning in the article, thus preventing students from wasting time looking for useless information. Secondly, guess the meaning of words based on the context of the original content. Any English word is not isolated in a sentence, so after a careful reading of the article, students can be encouraged to guess the meaning of the word according to the context and the clues, but logic of the guessing must be guaranteed. Thirdly, conduct guessing by the word-formation method. Generally, there are three methods of word formation, which are compound, transformation, and derivative. The root is the basis of a word formation. Mastering the common roots, prefixes and suffixes is of great necessity. By means of word formation, guessing the meaning will be possible. Finally, the contrast relations can also be used to guess the meaning. When there is a conjunction in the middle, we can guess the meaning of the word by using the contrasting relationship, which can effectively achieve the goal ultimately. 


\section{Conclusion}

Fostering students' English reading capabilities is a long process, in which perseverance and patience are required for gradual improvement. While conducting the actual teaching of English in vocational colleges, teachers should take the teaching content and the actual situation of students into consideration, adopt some targeted and personalized reading instructional strategies, and impart certain reading skills and methods to students, as well as spark students' enthusiasm and interest in reading English, and create opportunities for students to read independently. Only by doing these, students' reading speed and efficiency can be gradually enhanced, and their English proficiency can be improved on this basis.

\section{References}

[1] Gao Liyan. Brief Discussion on Cultivating Students' Interest in Learning English in Classroom Teaching [J]. Hua Zhang. Volume 21, 2011

[2] He Bo. Attention on Stimulating Students' Interest in English Teaching [J]. Heilongjiang Science and Technology Information. Volume 22, 2011

[3] Ma Yunxiang. How to Stimulate and Cultivate Interest in English Learning in the New Curriculum Reform [J]. The Science Education Article Collects (the first periodical appearing once every ten days); Volume 05, 2007

[4] Xie Lin. Brief Discussion on Stimulating Students' Interest in Learning English by Lively and Vivid Teaching Methods [J]. China Education Innovation Heral. Volume 21, 2009 